\title{
CORRELATION OF VISUAL ROUGHNESS PARAMETERS WITH THE AVERAGE VALUE OBTAINED FROM AISI 347 STAINLESS STEEL
}

\author{
SIR-ALEXCI SUAREZ ${ }^{1}$, ALBERT MIYER SUAREZ ${ }^{2}$ \& KARIME HERNÁNDEZ GEGEN ${ }^{3}$ \\ ${ }^{1}$ Engineering Faculty, University Francisco of Paula Santander Ocaña, Colombia \\ ${ }^{2,3}$ Faculty of Engineering and Architecture. University of Pamplona, Colombia
}

\begin{abstract}
This article presents a method that allows to correlate descriptors obtained through the artificial vision techniques of the co-occurrence matrix and Laws, in order to find the best descriptor that allows a measurement of roughness in AISI 347 stainless steel surfaces, similar to the value obtained using a contact profilometer. It was found that by combining the Laws descriptors they allow a better correlation by changing the kernel core that allows to measure curls with an accuracy of more than 92\%, this will allow it to be used to quantify the roughness without having contact with the surface, increasing the manufacturing process of parts in inline production systems.
\end{abstract}

KEYWORDS: Biomass, Forest biomass, wood energy, renewable energy, logistics, power generation

Received: Nov 20, 2020; Accepted: Dec 11, 2020; Published: Dec 22, 2020; Paper Id.: IJMPERDDEC202047

\section{INTRODUCTION}

The visual parameters obtained from the statistical descriptors of the images are constantly studied in order to find the descriptor that best correlates with the mean value of surface roughness $(\mathrm{Ra})$, the advantage of these visual parameters are its efficiency when it is implemented in factories that maintain a daily production line (Urriolagoitia-Sosa et al., 2012), where the different stages are concatenated for the production of the products (Zuno et al., 2017). The importance of roughness in the final product is evidenced by its quality and its origin can be determined from machining (Calvo-Correa et al., 2017; Morales Tamayo et al., 2014).

Roughness not only determines the visual appearance of a part, it is also important in its performance and duration that can affect the surfaces where they must be located for operation. The contact technique based on the probe uses $\mathrm{Ra}$ as the main parameter that is why the non-contact techniques that use artificial vision try to correlate the statistical descriptors with this parameter. Among the works developed is the arithmetic average or the average of the image $(\mathrm{Ga})$, calculated by counting the values present in the histogram (Bose \& Kumar, 2016; Kumar et al., 2005), but its value is not always exact, that is why investigations have been carried out where the color of the images is used (Lu et al., 2018; Yi et al., 2016) obtaining results with 90\% precision. An attempt is also made to acquire the images in 3 dimensions by varying the lighting (Zhongxiang et al., 2009) and correlating more parameters to increase effectiveness. The configuration of the vision systems can cause a distortion in the results as an example if the surface is not kept horizontal the results will be low, even if the processing techniques are adequate (Priya \& Ramamoorthy, 2007). The treatment of the image requires in most cases methods to improve the image, which are divided into the spatial and frequency domain, the first is done by quantifying the pixels directly, while the second makes some modifications through transformed as Fourier (Bethencourt et al., 1998) or Wavelet 
(Cerda et al., 2006). Within the frequency domain there are methods that use the co-occurrence matrix (Alves et al., 2010) and the local binary pattern (LBP), among others, using the texture present in the images.

In this work a correlation between descriptors is carried out using the Laws kernel and the correlation descriptor of the co-occurrence matrix in order to know which one can be more exact with the Ra values previously taken with the profilometer, this will allow to strengthen the visual parameters already studied such as Ga obtained from the grayscale image.

\section{METHODOLOGY}

The experiments were carried out with 3801 images with a resolution of $3272 \times 2469$ pixels, grouped into two classes according to the roughness value and trying to divide the number of images into the two classes in equal ways. The high class consists of 1,961 images with a roughness greater than $4 \mu \mathrm{m}$, the low class with 1840 images with a roughness less than $4 \mu \mathrm{m}$. At the moment it was only classified by comparing the texture values with those obtained by the Ra parameter of the roughness meter, 3 values were taken and averaged to obtain a more reliable roughness value. A vector of 9 characteristics is created and the Laws statistics are correlated (Laws, 1980) with a 9-feature vector with L5 - E5 - S5 - R5 - W5. And co-occurrence matrix (GLCM) with distance from 1 to 5 and 4 degrees from 0 to 90 . The images were acquired from the Research group in Science and Technology of the Francisco de Paula Santander Ocaña University, using stainless steel: AISI 347 (Fe / Cr18 / Ni10 / Nb) (Figure 1).

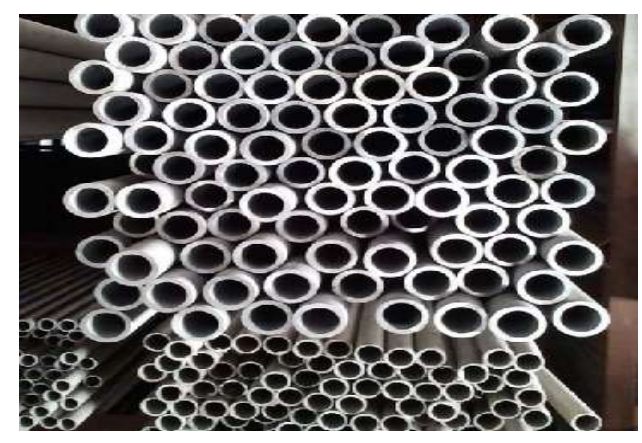

Figure 1: AISI 347 steel (Fe / Cr18 / Ni10 / Nb).

To use Laws kernels, the convolution process was carried out as shown in Figure 2 where each value varies according to the selected kernel. For the co-occurrence matrix, the normalized equation (equation 1) is used and the statistical correlation descriptor is obtained (equation 2).

$$
\begin{gathered}
N_{d}(i, j)=\frac{\operatorname{matrix}_{d}(i, j)}{\sum_{i} \sum_{j} \operatorname{matrix}_{d}(i, j)} \\
\text { Correlación }=\frac{1}{\sigma_{i} \sigma_{j}}\left|\sum_{i} \sum_{j}\left(i-u_{i}\right)\left(j-u_{j}\right) C(i, j)\right|
\end{gathered}
$$

With the deviation and the mean of $i, j$ 


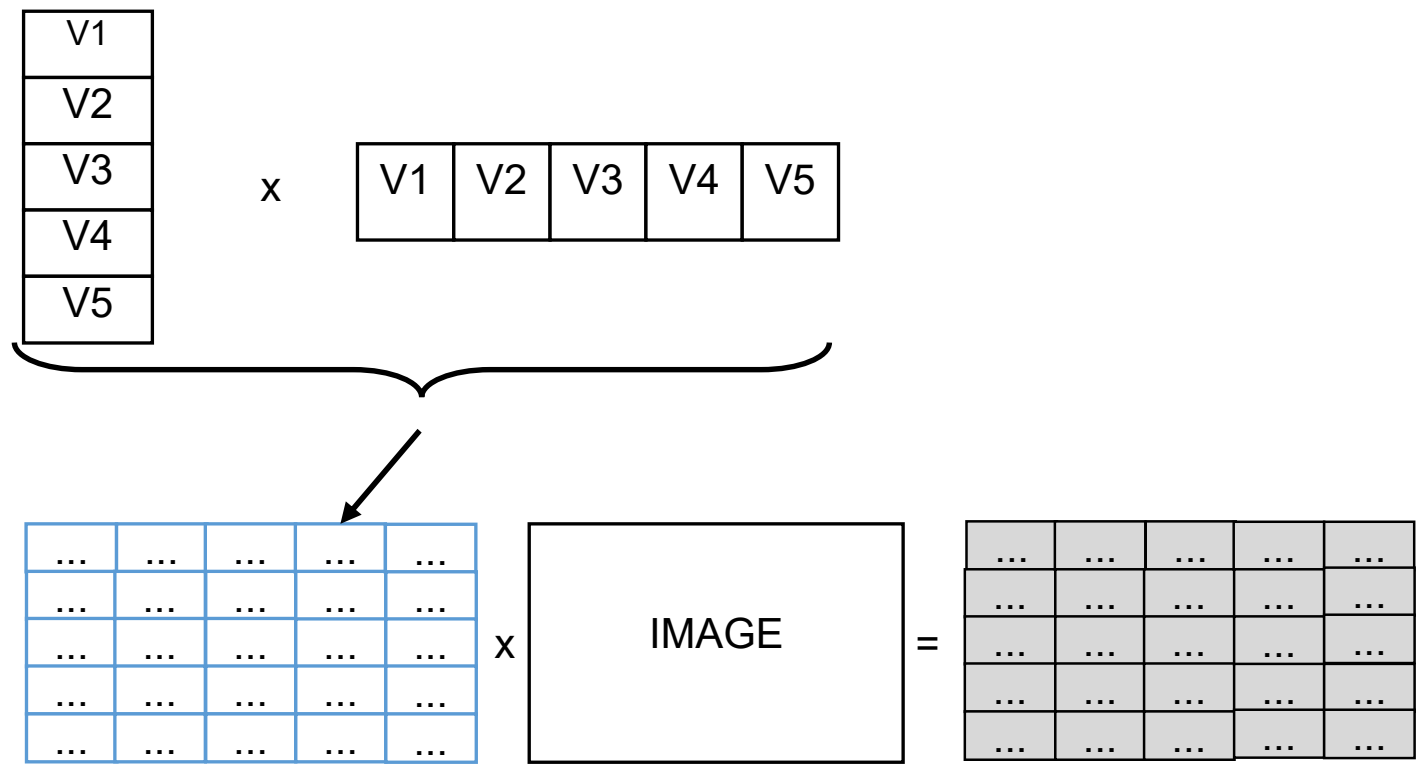

Figure 2: Laws convolution process

\section{RESULTS}

In table 1, we can observe the results with the Laws descriptors. When comparing the number of samples in the two classes, we verify an equality between the badly classified samples, maintaining a difference of $1 \%$. As $\mathrm{K}$ increases, an increase in error is observed in class 1 , this is due to the fact that there is little difference between the roughness values found in the border of the two classes, however class two remains almost invariant due to that there are higher values of roughness in these samples $(4 \mu \mathrm{m}-21 \mu \mathrm{m})$.

Table 1: Evaluation with Laws

\begin{tabular}{|c|c|c|c|}
\hline $\mathbf{K}$ & \% Class 1 error & \% Class 2 error & \% Error \\
\hline 1 & 11.52 & 10.45 & 10.97 \\
\hline 3 & 11.84 & 09.79 & 10.78 \\
\hline 5 & 13.04 & 10.50 & 11.73 \\
\hline 7 & 13.47 & 10.60 & 11.99 \\
\hline 9 & 14.02 & 10.60 & 12.25 \\
\hline 11 & 14.51 & 10.04 & 12.20 \\
\hline 13 & 15.05 & 10.24 & 12.57 \\
\hline 15 & 15.54 & 10.40 & 12.89 \\
\hline 17 & 15.86 & 10.14 & 12.91 \\
\hline 19 & 16.14 & 10.04 & 12.99 \\
\hline 21 & 16.19 & 9.89 & 12.94 \\
\hline 23 & 16.63 & 9.68 & 13.04 \\
\hline
\end{tabular}

In table 2Error! Reference source not found., the results obtained in the evaluations for the co-occurrence matrix with $0^{\circ}$ in distances of 1,2 and 3 are shown, using the thirteen descriptors. It is observed that the data are similar to those obtained with Laws but slightly increase the classification error by $0.45 \%$. It is also worth noting the importance of 
testing with different angles in order to know the invariant rotation of the texture based on the roughness of the materials.

Table 2: GLCM evaluation

\begin{tabular}{|c|c|c|c|}
\hline K & \% Class 1 error & \% Class 2 error & \% Error \\
\hline 1 & 11.79 & 11.32 & 11.54 \\
\hline 3 & 13.09 & 10.55 & 11.78 \\
\hline 5 & 13.15 & 9.38 & 11.20 \\
\hline 7 & 13.69 & 9.89 & 11.73 \\
\hline 9 & 14.07 & 9.43 & 11.68 \\
\hline 11 & 14.34 & 9.38 & 11.78 \\
\hline 13 & 14.23 & 10.24 & 12.18 \\
\hline 15 & 14.18 & 10.24 & 12.15 \\
\hline 17 & 14.61 & 10.91 & 12.70 \\
\hline 19 & 14.23 & 11.93 & 13.04 \\
\hline 21 & 14.67 & 12.79 & 13.70 \\
\hline 2.3 & 14.61 & 13.00 & 13.78 \\
\hline
\end{tabular}

When verifying the results of all the tests with the GLCM, it was observed that they did not improve discrimination, but neither did they increase the error too much, therefore it can be said that specifically in this type of material, rotation is not a significant problem.

Summarizing the best results for Laws it was $10.78 \%$ error, GLCM $0^{\circ}$ error of $11.20 \%$, GLCM $45^{\circ}$ error of $11.36 \%$, GLCM $90^{\circ}$ error of $11.31 \%$, GLCM $135^{\circ}$ error of $11.39 \%$.

In Figure 3, the errors of the descriptors evaluated between Laws and GLCM can be seen. Whose results showed a similar performance with a difference of $0.45 \%$ error in the classification, however the process time favorable to Laws was taken into account.

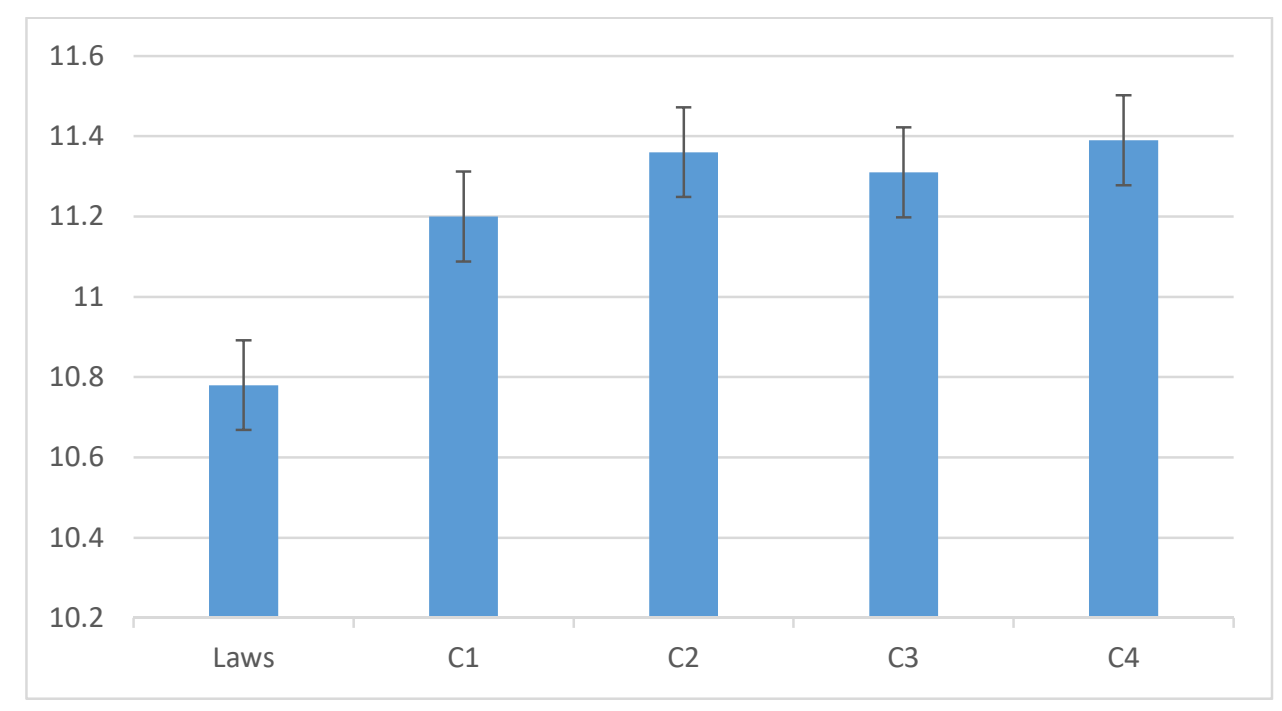

Figure 3: Evaluation between the Laws and GLCM descriptors.

Laws with a 9-feature vector with L5 - E5 - S5 - R5 - W5. Co-occurrence matrix (C1, C2, C3, C4) from 1 to 5 and with the 4 degrees. 


\section{Evaluation of Laws Convolution Kernels}

When determining the results and evaluating them with previous tests, we proceeded to determine which of the Laws masks performed a better solution to measure the roughness of the images. In order to evaluate only those kernels that presented optimal performance when characterizing the image based on the roughness of the texture, three Laws masks were selected, generated mainly from the R5 kernel, creating three two-dimensional kernels (R5R5, E5E5, R5E5). K-nn and the descriptors were used as classifier: mean, standard deviation, kurtosis and skewness.

In Figure 4, the results obtained in this phase are displayed, showing that the kernel obtained from R5R5 is the one that presents a lower error rate in said classification. Therefore, it was expected since said kernel is proposed to characterize the roughness in the images. Although it is observed that the global error increases because only one 2D convolution kernel is used, while in the previous phase nine are used. So based on these results it was decided to increase the discrimination power of said kernel (R5R5).

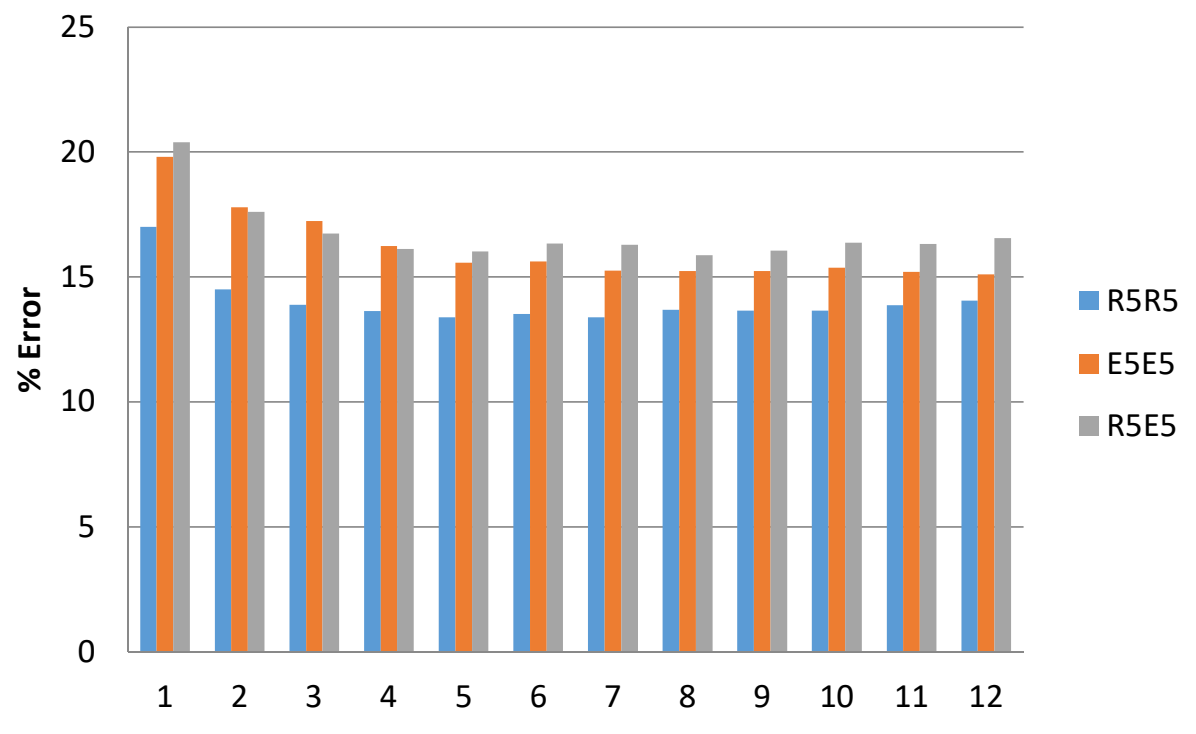

Figure 4: Classification by k-nn with three types of Laws kernel.

In this phase the important thing was to improve the classification results obtained, the proposal of this kernel consisted of improving the classification error starting from the maximum error base of $10 \%$, then any proposal greater than this value was not taken into account, although yes analyzed. To solve the problem, some weak points in the Laws kernels were checked. The difference consisted in changing the kernel in some cases, in others the whole kernel. And finally increase the neighborhood of the R5 kernel or by varying the two previous steps. In such a way that three rings were created which could vary when performing the convolution. Then six tests were carried out with each new 2D kernel, convolving with the image following the same Laws process. Both the number of images, configuration,

Table 3: Changing the core of $R 5$

\begin{tabular}{|c|c|c|c|}
\hline $\mathbf{K}$ & \% Class 1 error & \% Class 2 error & \% Error \\
\hline 1 & 12.22 & 11.11 & 11.65 \\
\hline 3 & 11.14 & 7.13 & 9.07 \\
\hline 5 & 11.79 & 6.52 & 9.07 \\
\hline
\end{tabular}




\begin{tabular}{|c|c|c|c|}
\hline 7 & 11.84 & 5.96 & 8.81 \\
\hline 9 & 11.52 & 5.76 & 7.99 \\
\hline 11 & 11.79 & 5.86 & 8.73 \\
\hline 13 & 11.73 & 6.17 & 8.86 \\
\hline 15 & 11.57 & 6.42 & 8.91 \\
\hline 17 & 12.22 & 6.17 & 9.10 \\
\hline 19 & 12.33 & 6.11 & 9.12 \\
\hline 21 & 12.39 & 6.27 & 9.23 \\
\hline 2.3 & 12.66 & 6.01 & 9.23 \\
\hline
\end{tabular}

Table 4 shows the best results obtained with the six Laws kernels and the correlation is higher than $92 \%$.

Table 4: Results with modified kernels

\begin{tabular}{|c|c|c|c|}
\hline $\mathbf{K}$ & \% Class 1 error & \% Class 2 error & \% Error \\
\hline 1 & 12.77 & 13.15 & 12.97 \\
\hline 3 & 11.52 & 10.75 & 11.12 \\
\hline 5 & 12.17 & 9.33 & 10.70 \\
\hline 7 & 11.90 & 9.07 & 10.44 \\
\hline 9 & 12.28 & 8.51 & 10.33 \\
\hline 11 & 12.11 & 8.51 & 10.26 \\
\hline 13 & 12.22 & 8.26 & 10.18 \\
\hline 15 & 12.39 & 8.10 & 10.18 \\
\hline 17 & 12.39 & 7.70 & 9.97 \\
\hline 19 & 12.22 & 8.00 & 10.01 \\
\hline 21 & 12.22 & 8.00 & 10.01 \\
\hline 2.3 & 12.33 & 8.00 & 10.10 \\
\hline
\end{tabular}

\section{CONCLUSIONS}

Products developed by all factories need a system that evaluate the value of roughness, which contact devices such as profilometer are generally used, and have some disadvantages for the same reason of their operation. Using artificial vision techniques increases the number of evaluations per product and reduces losses that could cause damage to the devices where the manufactured parts are assembled. The results allow to know visual descriptors that can be used as parameters similar to the Ra value of the profilometer, obtaining results that can be used for development in the production line. By combining several descriptors the correlation increases considerably and is one of the ways that they can be used.

\section{REFERENCES}

1. Alves, M., Ferreira, B., \& Leta, F. (2010). Evaluation of Roughness Parameters using Image Analysis from Different Optical and Electronic Microscopes. Technological information, 22, 129-146. https://doi.org/10.4067/S0718-07642011000400014

2. Bethencourt, M., Botana, J., Calvino, JJ, Marcos-Bárcena, M., \& Rodríguez-Chacón, M. (1998). Application of Fourier analysis to the study of roughness profiles of eroded samples. Revista de Metalurgia, 34. https://doi.org/10.3989/revmetalm.1998.v34.iExtra.698 
3. Bose, N., \& Kumar, DP (2016). Non-contact Surface Roughness Measurement of Engineering surface by the application of digital image magnification. International Journal of Fracture and Damage Mechanics, 1 (1), 01-11.

4. Calvo-Correa, MP, Benitez-Forero, JA, \& Cortés-Rodríguez, CJ (2017). Surface roughness analysis (RA) in the machining of Poly-Ether-Ether-Ketone (PEEK) for application in individualized implants. Mexican Journal of Biomedical Engineering, 38 (3), 517-523.

5. Cerda, E., Correa, JJ, \& Brambila, OG (2006). Application of the Wavelet transform in the detection of defects caused by vibration in rectified cylindrical parts. Undefined. / paper / Aplicaci\% C3\% B3n-de-la-Transformada-Wavelet-en-la-de-porCerda-Correa / f31083c675730684abc31a131f5fe38809f881b9

6. Kumar, R., Kulashekar, P., Dhanasekar, B., \& Ramamoorthy, B. (2005). Application of digital image magnification for surface roughness evaluation using machine vision. International Journal of Machine Tools and Manufacture, 45, $228-234$. https://doi.org/10.1016/j.ijmachtools.2004.07.001

7. Laws, K. (1980). Textured image segmentation. Ph. D: University of Southern California.

8. Lu, E., Liu, J., Rongyu, G., Yi, H., Wang, W., \& Suo, X. (2018). Designing indices to measure surface roughness based on the color distribution statistical matrix (CDSM). Tribology International, 122. https://doi.org/10.1016/j.triboint.2018.02.033

9. Morales Tamayo, Y., Pérez Rodríguez, R., Zambrano Robledo, P., Ávila Rondón, R., \& Zamora Hernández, Y. (2014). Experimental study of the surface roughness in the high speed dry turning of AISI $316 \mathrm{~L}$ steel destined for the food industry. Agricultural Technical Sciences Magazine, 23 (3), 54-59.

10. Priya, P., \& Ramamoorthy, B. (2007). The influence of component inclination on surface finish evaluation using digital image processing. International Journal of Machine Tools and Manufacture, 47, 570-579. https://doi.org/10.1016/j.ijmachtools.2006.05.005

11. Urriolagoitia-Sosa, G., Guerrero-Rivera, M., Romero-Ángeles, B., Hernández-Gómez, LH, Torres-San Miguel, CR, \& Urriolagoitia-Calderón, G. (2012). Methodology for the manufacture of components in 304 stainless steel through the cold forming process. Technical Journal of the Faculty of Engineering Universidad del Zulia, 35 (3), 243-251.

12. Yi, H., Liu, J., Ao, P., Lu, E., \& Zhang, H. (2016). Visual method for measuring the roughness of a grinding piece based on color indices. Optics Express, 24 (15), 17215-17233. https://doi.org/10.1364/OE.24.017215

13. Zhongxiang, H., Lei, Z., Jiaxu, T., Xuehong, M., \& Xiaojun, S. (2009). Evaluation of three-dimensional surface roughness parameters based on digital image processing. International Journal of Advanced Manufacturing Technology, 40, $342-348$. https://doi.org/10.1007/s00170-007-1357-5

14. Zuno, J., López, I., Granillo, M., Guerra, FV, \& Vera Gomez, U. (2017). Analysis of advanced steels for the manufacture of crankshafts used in turbo engines. Ingenio y Consciencia Scientific Bulletin of the Higher School of Cd. Sahagún, 4. https://doi.org/10.29057/ess.v4i7.2053 

